\title{
A Computational Method of Radar Jamming Cover Area for Aerial Platform Radar Countermeasures
}

\author{
Honghao Zuo ${ }^{1, a}$ \\ ${ }^{1}$ Electronic Engineering Institute, Hefei, 230037, China \\ aemail: zuohefei@tom.com
}

Keywords: Aerial Platform; Radar Jamming; Radar Jamming Cover Area

\begin{abstract}
There are more meaning of researching the operational efficiency of aerial radar jamming equipments and the method of using them than ever while the new aerial equipments have been widely used in air force. A novel computational method of Radar Jamming cover area is proposed in this paper, which is different from two traditional ways: exposure area method and radar's detection coverage method. Considering the different spatial relationship among the radar jammer, the cover area and the target radar, which is formed in the situation of air to air jamming and air to ground jamming, the computational method of aerial platform radar jamming cover area based on basic radar equation is given. The simulation experiments testify the new method is effective.
\end{abstract}

\section{Introduction}

Radar jamming and deception is the intentional emission of radio frequency signals to interfere with the operation of a radar by saturating its receiver with noise or false information [1][2]. Conventional radar jammer implements its combat operation by two ways, ground to ground and ground to air. Generally there are two ways to research the radar jammer's combat effectiveness. One is the exposure area of the covered target [3][4]. The other is radar detection coverage after being jammed [5]. A third way of radar jamming cover area (RJCA below) is proposed by author [6]. The computation method has some limitation in the case because ground to air modal is the only combat background that is considered in the paper. Nowadays, more and more aerial platform radar jammers are available in military troops, such as electronic warfare support aircraft, electronic warfare UAV, and airborne jammer nacelle and so on. It is important to help the air force commanders to deploy the power effectively.

It is arranged below: In Section II the definition and meaning of RJCA is introduced. Considering the different spatial relationship among the radar jammer, the cover area and the target radar, which is formed in the situation of air to air jamming and air to ground jamming, the computational method of aerial platform radar jamming cover area is given in Section III. In Section IV the simulation experiment results including sectional views of horizontal and vertical are given. In the end the conclusion is given.

\section{Radar Jamming Cover Area}

RJCA is a new term for the moment and its definition hasn't been given out by authority. It was firstly introduced by Luo and Li [6]. Radar jamming cover area is the three-dimensional space, where the covered target can't be detected normally by the specified radar when radar countermeasures are running [7]. The RJCA is a diorama surrounding the jammer. The RJCA varies with different distance between jammer and radar and their parameters. The RJCA which joint operations commanders concerned in ground to air defense operation is a plane on the surface, while the RJCA which air force commanders concerned in air-to-air combat or air-to-ground combat is a three Dimension surrounding the jammer. Its concerning field expands from plane to $3 \mathrm{D}$.

The RJCA varies according to the different spatial relationship among the radar jammer, the cover area and the target radar. There are some laws when the parameters vary. If there is only one 
variable in all parameters, the power of jammer is bigger, the size of RJCA is bigger. If the location of radar, jammer is constant, the radar cross section (RCS) of the covered target is bigger, the size of RJCA is smaller. If the RCS of the covered target is constant, the distance between radar and jammer is farer, the size of RJCA is bigger.

Although the tactical background of RJCA varies, the computational principle of RJCA is constant. Keeping the wholeness of the article, the computational principle of RJCA is repeated as below which is excerpted from [7].

Considering air-to-air and air-to-ground model, the computational principle of radar jamming cover area can be made as below.

It is assumed that radar antenna is aiming at the covered target and the vertical plane passing through the radar antenna and the covered target is a symmetrical plane on antenna pattern.

Based on radar equation, calculation method of RJCA is given as (1):

$\forall$ point $C \in\{$ RJCA $\}$, it should satisfy:

$\frac{P_{r j}}{P_{r s}} \geq K$

where, $\mathrm{P}_{\mathrm{rs}}$ is the power radar received from the target reflecting; $\mathrm{P}_{\mathrm{rj}}$ is the power radar received from the jammer emitting; $\mathrm{K}$ is a suppression coefficient assigned by user in different situation.

From the basic radar equation, it can be concluded as below:

$$
P_{r s}=\frac{P_{t} G_{t} \sigma A}{\left(4 \pi R_{t}^{2}\right)^{2}}=\frac{P_{t} G_{t} \sigma \lambda^{2}}{(4 \pi)^{3} R_{t}^{4}}
$$

where, $P_{t} G_{t}$ is radar equivalent radiation power; $\sigma$ is RCS of the target; $R_{t}$ is the distance between radar and the target; $A$ is radar antenna equivalent receive area; $\lambda$ is radar working wavelength.

$$
P_{r j}=\frac{P_{j} G_{j} G_{t}^{\prime}\left(\theta_{1}, \varphi_{1}\right) \lambda^{2} r_{j}}{\left(4 \pi R_{j}\right)^{2}}
$$

where, $P_{j} G_{j}$ is jammer's equivalent jamming power; $R_{j}$ is the distance between radar and the jammer; $\mathrm{r}_{\mathrm{j}}$ is polarization loss; $G_{t}^{\prime}\left(\theta_{1}, \varphi_{1}\right)$ is radar antenna gain at direction of aiming jammer.

From (1),(2),(3), we can get:

$$
\frac{P_{j} G_{j}}{P_{t} G_{t}} \cdot \frac{4 \pi r_{j}}{\sigma} \cdot \frac{G_{t}^{\prime}\left(\theta_{1}, \varphi_{1}\right)}{G_{t}} \cdot \frac{R_{t}^{4}}{R_{j}^{2}} \geq K_{j}
$$

In (4) all parameters value is given except $G_{t}^{\prime}\left(\theta_{1}, \varphi_{1}\right)$. The radar array antenna model is given as below:

$$
G\left(\theta_{1}, \varphi_{1}\right)=G_{0} \sin c^{2}\left(\frac{M d_{x} \pi}{\lambda} \sin \theta_{1}\right) \cdot \sin c^{2}\left(\frac{N d_{y} \pi}{\lambda} \sin \varphi_{1}\right)
$$

where, $\mathrm{G}_{0}$ is radar main lobe gain; $\mathrm{M}$ is the number of columns of array antenna; $\mathrm{N}$ is the number of rows of array antenna; $d_{x}$ is the distance between array column elements; $d_{y}$ is the distance between array row elements; $\theta_{1}$ is the angle between radar beam and the horizontal plane; $\varphi_{1}$ is the angle between radar beam and the vertical plane.

\section{Computational Method of RJCA For Aerial Platform}

It can be derived from II.B that the core of RJCA computation is how to calculate radar antenna gain at direction of aiming jammer. Its computational method is different from that of ground to air model because of different spatial relationship.

The air-to-air model is shown in Fig.1. Plane $A_{B} C_{1}$ is a horizontal where jammer A located. We project $B, C$ by vertical projection to plane $A B_{1} C_{1}$ joining $B_{1}$ and $C_{1}$. It is assumed that the center of radar antenna beam is aiming at the covered target $C$. If not considering the aerial platform 
rolling around its body coordinate $\mathrm{X}_{\mathrm{b}}$, the vertical plane of radar beam is coincidence with plane $\mathrm{BCB}_{1}$, and is perpendicular to plane $\mathrm{AB}_{1} \mathrm{C}_{1}$. We draw a line through $\mathrm{A}$ perpendicular to plane $\mathrm{BCB}_{1}$ joining $\mathrm{A}_{\phi}$. Then the angle between jammer beam $\mathrm{AB}$ and vertical plane $\mathrm{BCB}_{1}$ is $\theta$. The angle between jammer beam $\mathrm{AB}$ and plane $\mathrm{AA}_{\phi} \mathrm{B}$ is $\phi . \theta=\angle A B A_{\phi}, \phi=\angle C B A_{\phi}$.

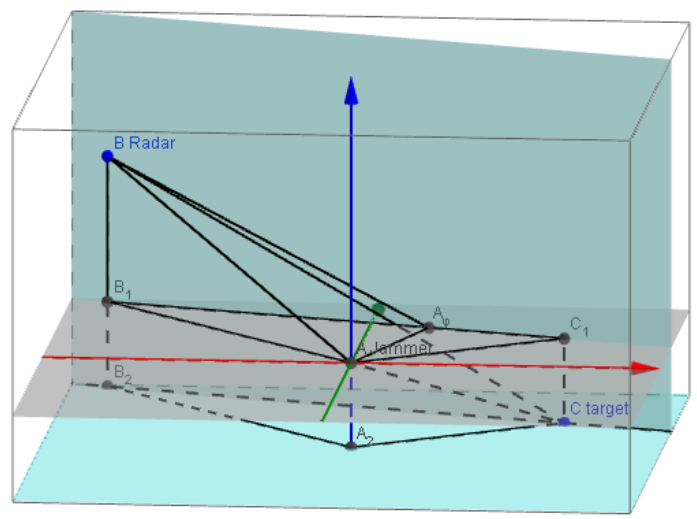

Fig.1.Radar height + , target height -

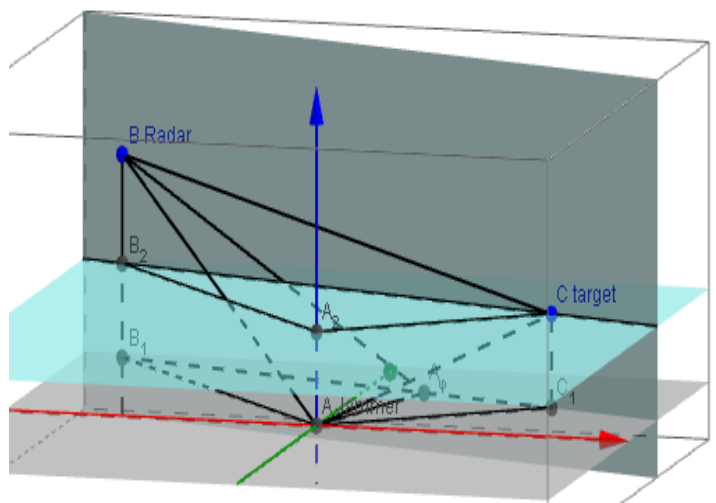

Fig.2 Radar height + , target height + $\overline{\mathrm{BB}_{1}}=\mathrm{H}, \quad \overline{\mathrm{CC}_{1}}=\mathrm{h}, \quad \overline{\mathrm{AB}}=\mathrm{R}_{\mathrm{j}}, \quad \overline{\mathrm{AB}_{1}}=\mathrm{R}_{\mathrm{j}}^{\prime}, \quad \overline{\mathrm{BC}}=\mathrm{R}_{\mathrm{t}}, \quad \overline{\mathrm{B}_{2} \mathrm{C}}=\mathrm{R}_{\mathrm{t}}^{\prime}, \quad \overline{\mathrm{AC}}=\mathrm{r}, \quad \overline{\mathrm{AC}_{1}}=\mathrm{r}^{\prime}$

$\mathrm{R}_{\mathrm{t}}^{2}=\mathrm{R}_{\mathrm{t}}^{\prime 2}+(\mathrm{H}+\mathrm{h})^{2}$

$\mathrm{R}_{\mathrm{j}}^{2}=\mathrm{R}_{\mathrm{j}}^{\prime 2}+\mathrm{H}^{2}$

$\angle \mathrm{AB}_{1} \mathrm{C}_{1}=\cos ^{-1}\left(\left(\overline{\mathrm{AB}}_{1}^{-2}+{\overline{\mathrm{C}_{1} \mathrm{~B}_{1}}}^{2}-\overline{\mathrm{AC}}_{1}^{-2}\right) /\left(2 * \overline{\mathrm{AB}_{1}} * \overline{\mathrm{C}_{1} \mathrm{~B}_{1}}\right)\right)$

$=\cos ^{-1}\left(\left(\mathrm{R}_{\mathrm{j}}^{\prime 2}+\mathrm{R}_{\mathrm{t}}^{\prime 2}-\mathrm{r}^{\prime 2}\right) /\left(2 * \mathrm{R}_{\mathrm{j}}^{\prime} * \mathrm{R}_{\mathrm{t}}^{\prime}\right)\right)$

$\overline{\mathrm{AA}_{\phi}}=\overline{\mathrm{AB}_{1}} * \sin \angle \mathrm{ABA} A_{\phi}=\mathrm{R}_{\mathrm{j}}^{\prime} * \sin \angle \mathrm{ABA}_{\phi}$

$\overline{\mathrm{B}_{1} \mathrm{~A}_{\phi}}=\overline{\mathrm{AB}_{1}} * \cos \angle \mathrm{ABA}_{\phi}=\mathrm{R}_{\mathrm{j}}^{\prime} * \cos \angle \mathrm{ABA}_{\phi}$

$\overline{\mathrm{BA}_{\phi}}=\left({\overline{\mathrm{BB}_{1}}}^{2}+{\overline{\mathrm{B}_{1} \mathrm{~A}_{\phi}}}^{2}\right)^{0.5}$

in $\triangle \mathrm{ABA}_{\phi}$ :

$\angle \mathrm{ABA}_{\phi}=\cos ^{-1}\left(\left(\overline{\mathrm{AB}}^{2}+\overline{\mathrm{BA}}_{\phi}^{2}-\overline{\mathrm{AA}}_{\phi}^{2}\right) /\left(2 * \overline{\mathrm{AB}} * \overline{\mathrm{BA}_{\phi}}\right)\right)$

$\overline{\mathrm{C}_{1} \mathrm{~A}_{\phi}}=\overline{\mathrm{C}_{1} \mathrm{~B}_{1}}-\overline{\mathrm{AA}_{\phi}}$

$\overline{\mathrm{CA}_{\phi}}=\left({\overline{\mathrm{CC}_{1}}}^{2}+{\overline{\mathrm{C}_{1} \mathrm{~A}_{\phi}}}^{2}\right)^{0.5}$

in $\triangle \mathrm{CBA}_{\phi}$ :

$\angle \mathrm{CBA}_{\phi}=\cos ^{-1}\left(\left(\overline{\mathrm{BC}}^{2}+\overline{\mathrm{BA}}_{\phi}^{2}-\overline{\mathrm{CA}}_{\phi}^{2}\right) /(2 * \overline{\mathrm{BC}} * \overline{\mathrm{BA}})\right)$

If the covered target is below the jammer, the diagram of spatial relationship among 3 objects, the jammer, the radar and the covered target is shown as Fig.2.

Compare to that of Fig.1, (6) turns into

$\mathrm{R}_{\mathrm{t}}^{2}=\mathrm{R}_{\mathrm{t}}^{22}+(\mathrm{H}-\mathrm{h})^{2}$

Equation (7) (15) are the same with that of Fig.1. 
When radar is located on the ground and the jammer is on the air, it's called air to ground model, which is illustrated in Fig.3.

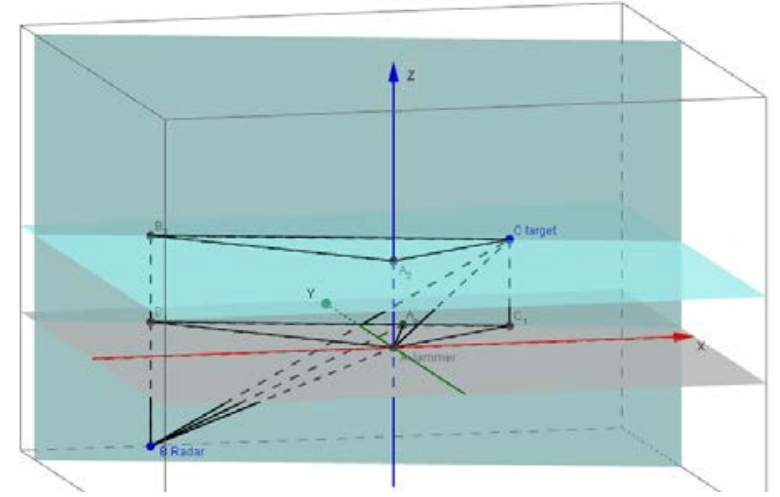

Fig.3. Air to ground model

The computational procedure is the same as that of air-to-air model. It is important to note that the height of radar in computation is a negative value.

\section{Experiment Results}

For convenience the radar in the experiment radar is a simulation of AN/APG-66(3), which has the phased array. To simulate a $1.5^{\circ} \times 3^{\circ}$ radar main lobe, the parameters of (5) are given as below: $\mathrm{M}=30 ; \mathrm{N}=24 ; \lambda=0.03 ; \mathrm{Dx}=0.01 ; \mathrm{Dy}=0.015$.

The simulation of antenna pattern is given as Fig. 4 and Fig.5.
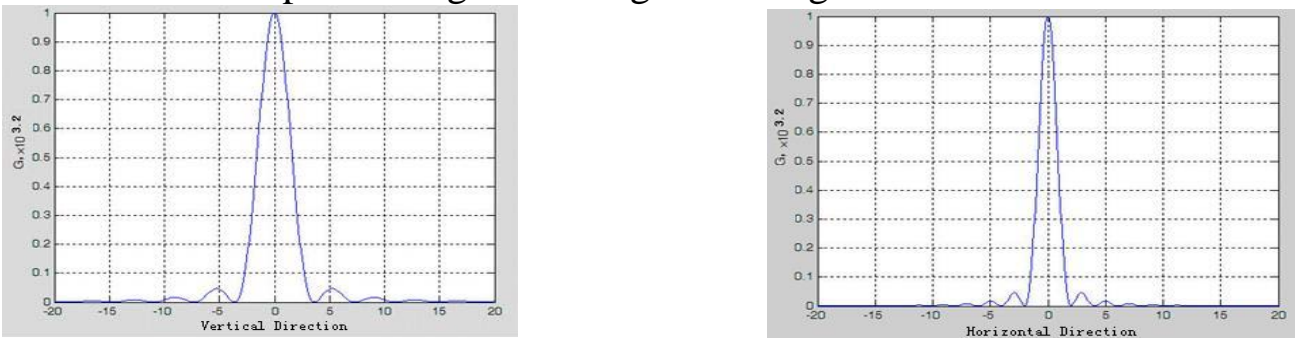

Fig.4.Vertical direction simulation of antenna Fig.5. Horizontal direction simulation of antenna

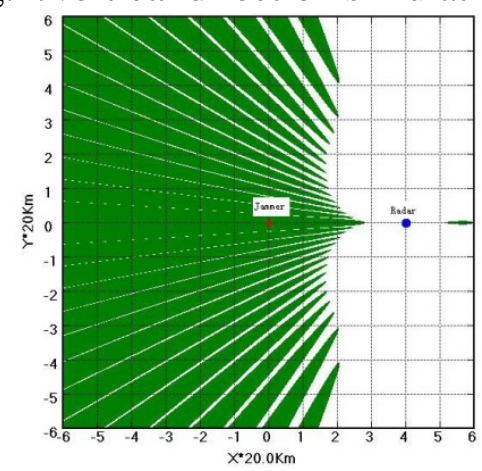

Fig.6. $\mathrm{H}=0 \mathrm{Km}$

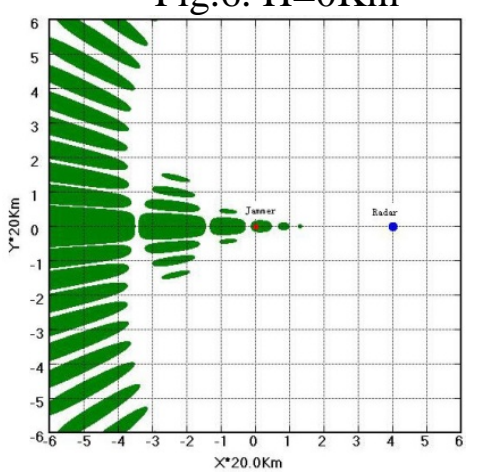

Fig.9. $\mathrm{H}=15 \mathrm{Km}$

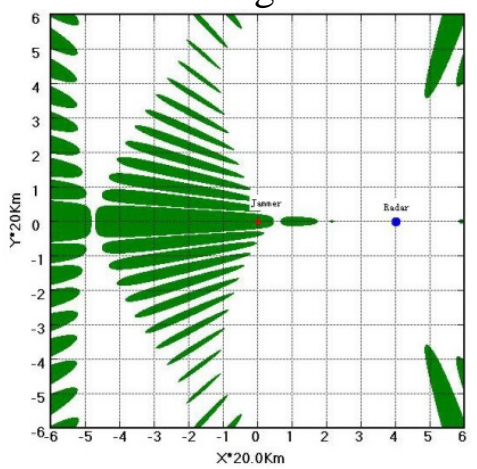

Fig.7. $\mathrm{H}=5 \mathrm{Km}$

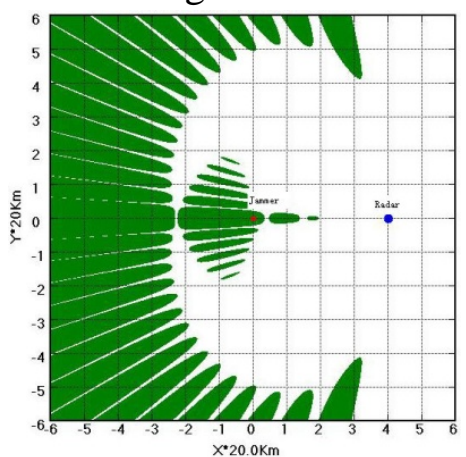

Fig.10. $\mathrm{H}=-5 \mathrm{Km}$

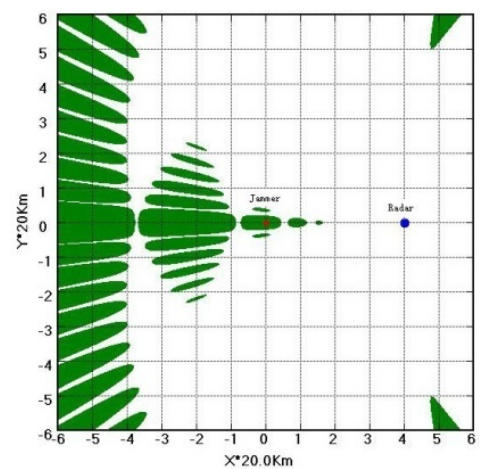

Fig.8. $\mathrm{H}=10 \mathrm{Km}$

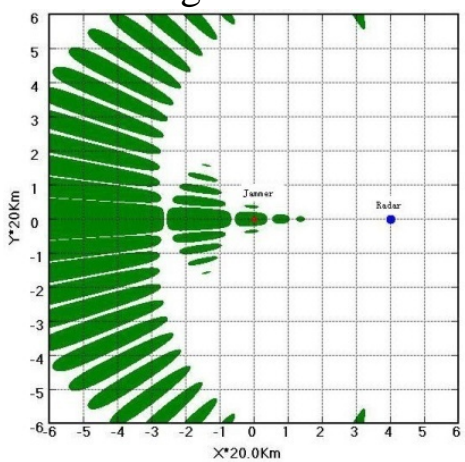

Fig.11. $\mathrm{H}=-10 \mathrm{Km}$ 
Fig.6 Fig.11 are the horizontal sections of RJCS.

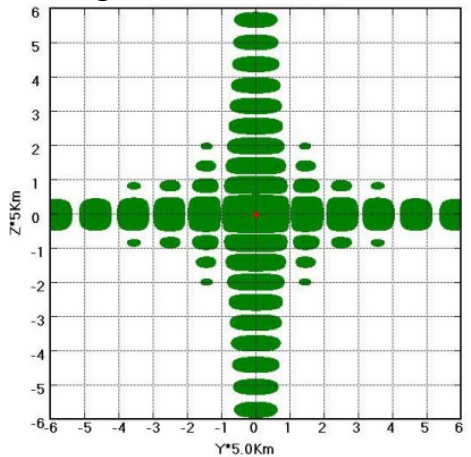

Fig.12. $\mathrm{X}=0 \mathrm{Km}$

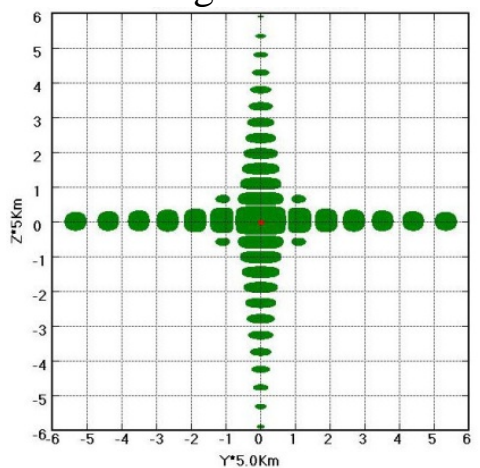

Fig.15. $X=20 \mathrm{Km}$

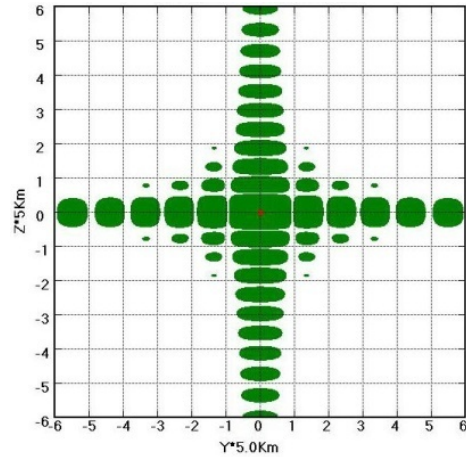

Fig.13. $X=5 \mathrm{Km}$

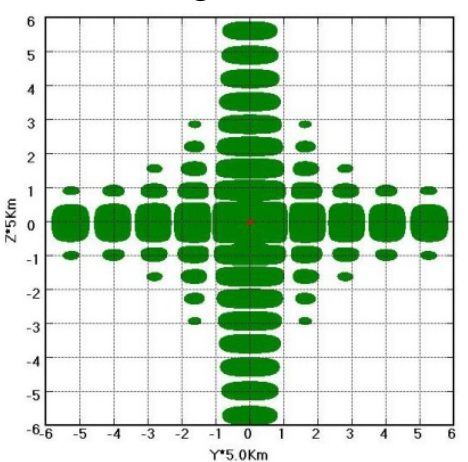

Fig.16. $X=-10 \mathrm{Km}$

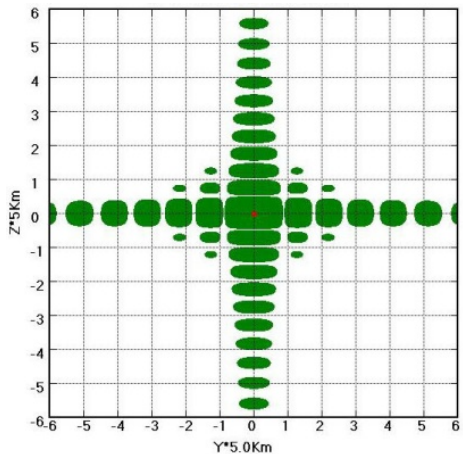

Fig.14. $X=10 \mathrm{Km}$

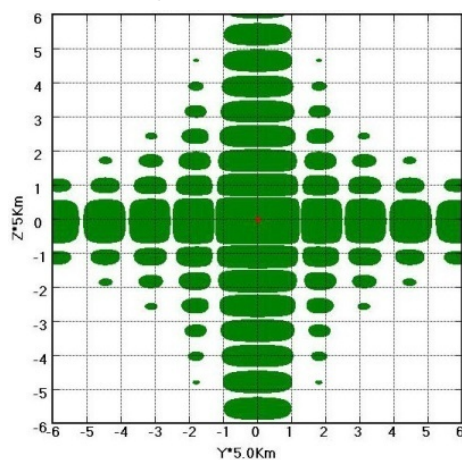

Fig.17. $X=-20 \mathrm{Km}$

Fig.12 Fig.17 are the vertical sections of RJCS parallel to YOZ.

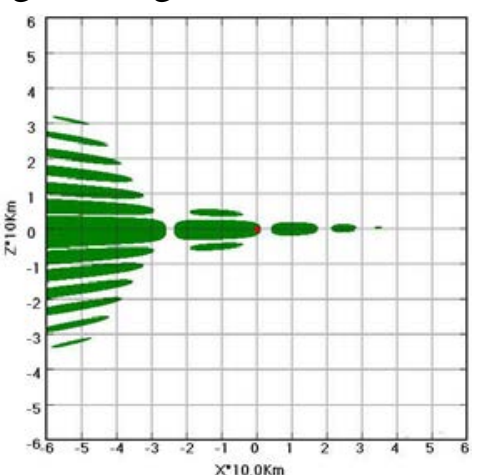

Fig.18. $Y=-20 \mathrm{Km}$

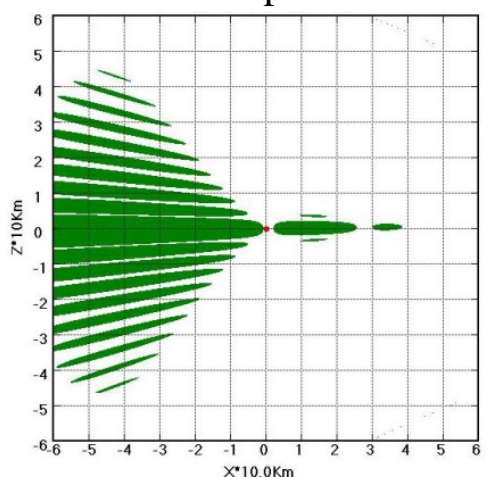

Fig.19. $Y=-10 \mathrm{Km}$

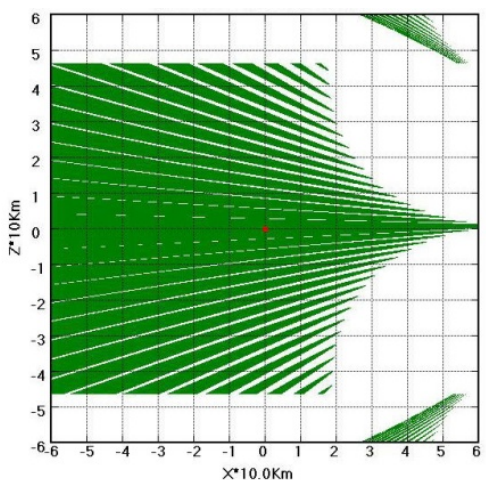

Fig.20. $\mathrm{Y}=0 \mathrm{Km}$

Fig.18 Fig.20 are the vertical sections of RJCS parallel to XOZ.

From the figures shown above we can construct the 3D structure of RJCS which is too complexity to be shown in a 2D image.

\section{Conclusion}

It can be concluded that RJCS is a domain centered the jammer and it is not continuous. In calculation process the jammer is set to the origin of the coordinate. By analyzing the series of RJCS section we can understand the radar jammer combat capability. It is helpful for the air force commanders to deploy the power effectively based on RJCS sections. The experiment results testify it's reasonable and effective.

\section{Acknowledgement}

Zuo H.H. thank my colleague Li Xiaoming, Zhang Y., Wei L.L., Zhu S.L. and Bi D.P. for discussing RJCS problem so much. 


\section{References}

[1] Website:https://en.m.wikipedia.org/wiki/radar_jamming_and_deception,april, 2016.

[2] Lin Xiangping. The principle of radar countermeasures. Xi'an: Northwest Telecommunication Engineering Institute Press, 1985.

[3] Shao Guopei, Cao Zhiyao, He Jun, et al. Electronic warfare combat effectiveness analysis. Beijing: People’s Liberation Army Press,1997.

[4] Shao Guopei, Electronic countermeasures tactical computing Method. Beijing: People’s Liberation Army Press,2010.

[5] Luo Guangcheng, Li Xiuhe and Li Xiaoming, A study on simulation and modeling of cover area for ground to air radar countermeasures. Modern Radar, 2014, 36(7):68-72.

[6] Zuo Honghao and Li Xiaoming, "A novel calculation method of radar jamming cover area for ground to air radar countermeasures”, 2016 IEEE information Technology, Networking, Electronic and Automation Control Conference, Chongqing, China, May 2016:897-900.

[7] Wang Guoshi, Li Qiang, Liu Yongpeng, et al. Calculation of detection range for AWACS patrol under condition of distributed interference. Modern Radar, 2013,35(10):70-75. 